\title{
Leaders
}

\section{Modern laboratory diagnosis of mycobacterial infections}

\author{
S A Watterson, F A Drobniewski
}

\begin{abstract}
This review summarises recent advances made in microscopic techniques (fluorescence and peptide nucleic acids) and culture techniques (solid, liquid, radiometric, and non-radiometric systems) and in the development of rapid methods for the identification of mycobacterial cultures (high performance liquid chromatography, thin layer chromatography, RNA sequencing, and polymerase chain reaction restriction enzyme assays). The role of molecular amplification systems in identifying Mycobacterium tuberculosis is described. Most methods record high specificity and sensitivity for smear positive sputum but have variable sensitivity for sputum smear negative and extrapulmonary specimens. Specimen quality will affect the performance of these assays and organisational delays, such as the batching of specimens, can reduce the time saved. In house assays can be as effective as commercial systems as long as appropriate controls are used. (F Clin Pathol 2000;53:727-732)
\end{abstract}

Keywords: tuberculosis; laboratory diagnosis; nontuberculous mycoplasma

For many years tuberculosis (TB) was thought to be conquered in the developed world. In the past decade, however, the incidence of TB in the UK has been rising. ${ }^{1}$ The World Health Organisation (WHO) has declared TB to be a global emergency, with approximately 3 million people dying of TB every year. ${ }^{2}$ This, and several high profile outbreaks of multidrug resistant TB (MDR-TB) in the UK and USA, has driven research into improved methods for the diagnosis of TB and other mycobacterial infections. Although of lesser public health importance than Mycobacterium tuberculosis, nontuberculous mycobacteria (NTM) have been isolated from patients in the UK with increasing frequency. This has been in part, but not solely, the result of increasing rates of human immunodeficiency virus (HIV) infection. ${ }^{3}$ There has been a pronounced reduction in the incidence of $M$ avium complex infections since the introduction of improved antiretroviral treatment.

Although empirical treatment regimens are used in the management of $M$ tuberculosis and NTM disease, drug susceptibility testing is particularly important in identifying drug resistance in TB, which is known to compromise therapeutic success. ${ }^{4}$ The identification of NTM species is necessary because this will determine which empirical strategy should be used. Drug susceptibility testing is of limited use for most NTM. ${ }^{5}$

This article will discuss new methodologies for the laboratory diagnosis of mycobacterial infections in the context of traditional methods and suggest appropriate clinical uses.

\section{The diagnostic mycobacteriology}

\section{laboratory}

The functions of the mycobacteriology laboratory vary depending on the population served, the incidence of $M$ tuberculosis and NTM disease, and whether the laboratory has a reference role. In the UK at the local level, the remit of the district laboratory is to examine clinical specimens for the presence of mycobacteria using microscopy and culture. However, many laboratories lack the resources to identify isolates or determine their drug susceptibilities. The reference laboratory exists to perform this vital task and in so doing produces disease surveillance data as part of the national tuberculosis programme.

The mainstays of the mycobacteriology laboratory are, and will remain for the foreseeable future, microscopy and culture on solid and in liquid media. Microscopy, although relatively insensitive, remains the only same day diagnostic test within the budget of all laboratories, large or small. In addition, because it assesses the infectivity of a patient with pulmonary tuberculosis, ${ }^{6}$ it is an indispensable test for hospital and community infection control. Conventional culture based techniques can take as long as six to eight weeks to isolate a mycobacterial species from a clinical specimen, but currently no other test is as sensitive. Of those tests that do approach the degree of sensitivity displayed by culture, none is capable of providing other essential data, such as the full range of drug susceptibilities. ${ }^{7}$ Laboratories must screen specimens rapidly for acid fast bacilli (AFB), and culture and identify isolates as quickly as possible. The United States Centres for Disease Control (CDC) have recommended the identification of $M t u$ berculosis cultures and the determination of first line drug susceptibilities to be performed within 30 days. $^{8}$ 
Advances in microscopy

Microscopy for mycobacteria has traditionally involved staining the specimen with carbol fuchsin dye, which is retained by the cell wall on washing with acid alcohol (for example, Ziehl-Neelsen; ZN). The time to examine a specimen by microscopy was shortened with the introduction of fluorescent microscopy and staining with auramine phenol. ${ }^{8}$ Proficiently performed microscopy can detect AFBs in $60-70 \%$ of culture positive respiratory specimens. ${ }^{9}$ The sensitivity of $\mathrm{ZN}$ microscopy is approximately $5 \times 10^{3} \mathrm{AFBs} / \mathrm{ml} .{ }^{10}$ Concentrating the sputum specimen can improve sensitivity, although some studies have found the reverse because not all mycobacteria sediment on centrifugation. ${ }^{11}$ Moreover, the unconcentrated method gives an accurate guide to the infectivity of the patient-patients with smear positive sputum are the most infectious. ${ }^{6}$ Microscopy on material from solid, and often liquid, cultures can be used to make a presumptive diagnosis of TB, although it is not possible to distinguish between $M$ tuberculosis and NTM in clinical specimens. ${ }^{8}$ However, a more accurate method (see below) is needed for identification at the species level.

The ability simultaneously to assess the smear status and distinguish between $M$ tuberculosis and NTM directly in a respiratory specimen has important implications for patient treatment and hospital infection control. A potential advance in microscopy that might facilitate this is the use of peptide nucleic acids (PNAs) in a fluorescent stain format. PNAs are DNA like molecules in which the sugar phosphate backbone is replaced with a peptide like structure. The binding of PNA to DNA or RNA is sequence specific and the interaction is stronger than that of a DNA-DNA interaction. Because of their hydrophobic nature, PNAs can pass more easily across intact cell walls, and with suitable treatment can bind to specific intracellular nucleic acid sequences. Labelling the PNA with a fluorescent dye enables visualisation with a suitable microscope. PNAs specific for the $M$ tuberculosis complex and NTM have been formulated and tested against a panel of cultured mycobacterial species with some success. ${ }^{12}$

\section{Advances in culture techniques}

Traditional culture is performed on solid and liquid media such as Löwenstein-Jensen, or Kirchner and the various Middlebrook formulations $(7 \mathrm{H} 9,7 \mathrm{H} 10$, and $7 \mathrm{H} 11)$. The time to detection of growth of a mycobacterial species can be shortened greatly by the use of an automated or semi-automated liquid culture system that can detect growth much earlier than the naked eye. The first such system to be used in the laboratory was the BACTEC 460TB instrument. Using a broth with radiolabelled ${ }^{14} \mathrm{C}$-palmitate as its sole carbon source, the metabolising organism converts this to radiolabelled ${ }^{14} \mathrm{CO}_{2}$, which can then be detected by the BACTEC instrument. New systems that rely on non-radiometric detection of growth have been developed, such as the MB/BacT (Organon Teknika), BACTEC 9000 (Becton
Dickinson), and the mycobacterial growth indicator tube (MGIT; Becton Dickinson). Many studies have compared the performance of the different culture systems available. The BACTEC 460 system remains the fastest and most sensitive, followed by the continuous automated non-radiometric liquid culture systems, with the solid media systems being the slowest. It is important to note that although a system may appear to be faster because its detection method is more sensitive, the biomass present in the culture bottle may be substantially less than that of the other systems. For example, the higher biomass of the $\mathrm{MB} / \mathrm{Bac} \mathrm{T}$ system allows direct inoculation of identification panels and susceptibility tests, and identification with DNA hybridisation systems in nearly all cases. Table 1 summarises some comparative evaluations of the different systems.

Most hospital laboratories in the UK rely solely on solid and/or manual liquid (for example, Kirchner) media for the isolation of mycobacteria ${ }^{19}$ because the workload involved does not make the purchase and use of an automated system a cost effective option. However, groups of laboratories acting together can make the purchase of such a system practical and, increasingly, many laboratories have chosen to do so. The PHLS MRU and the Regional Centres for Mycobacteriology can provide rapid culture services to hospitals for which they do not normally provide primary isolation services.

\section{Rapid methods of identification of mycobacteria}

Traditional methods are based upon growth parameters, biochemical characteristics, and the analysis of cell wall lipids by thin layer chromatography (TLC). Several systems exist for the rapid identification of mycobacterial species from cultured isolates. The Accuprobe (GenProbe, USA) system includes tests for the identification of the $M$ tuberculosis complex, $M$ avium complex, $M$ avium, $M$ intracellulare, $M$ kansasii, and $M$ gordonae. The system is highly specific but a separate test must be performed for each species. High performance liquid chromatography (HPLC) analysis of mycolic acids, ${ }^{20}$ and DNA sequencing of the $16 \mathrm{~S}$ ribosomal RNA (rRNA) gene ${ }^{21}$ can both be used for the reliable identification of mycobacterial species. However, both assays use high cost capital equipment beyond the reach of most clinical laboratories except at the reference level, although the low reagent costs of HPLC can offset the high set up costs. Another option is the polymerase chain reaction (PCR) restriction enzyme assay (PRA), ${ }^{22}$ which has been used with success in our laboratory and elsewhere. The assay is based on the PCR amplification of a fragment of the hsp65 gene. The PCR product is subsequently digested in two separate restriction enzyme digestions and the fragments analysed by agarose gel electrophoresis. The most recent algorithm as proposed by Devallois et al can be used to identify 34 mycobacterial species and subspecies. ${ }^{23}$ 


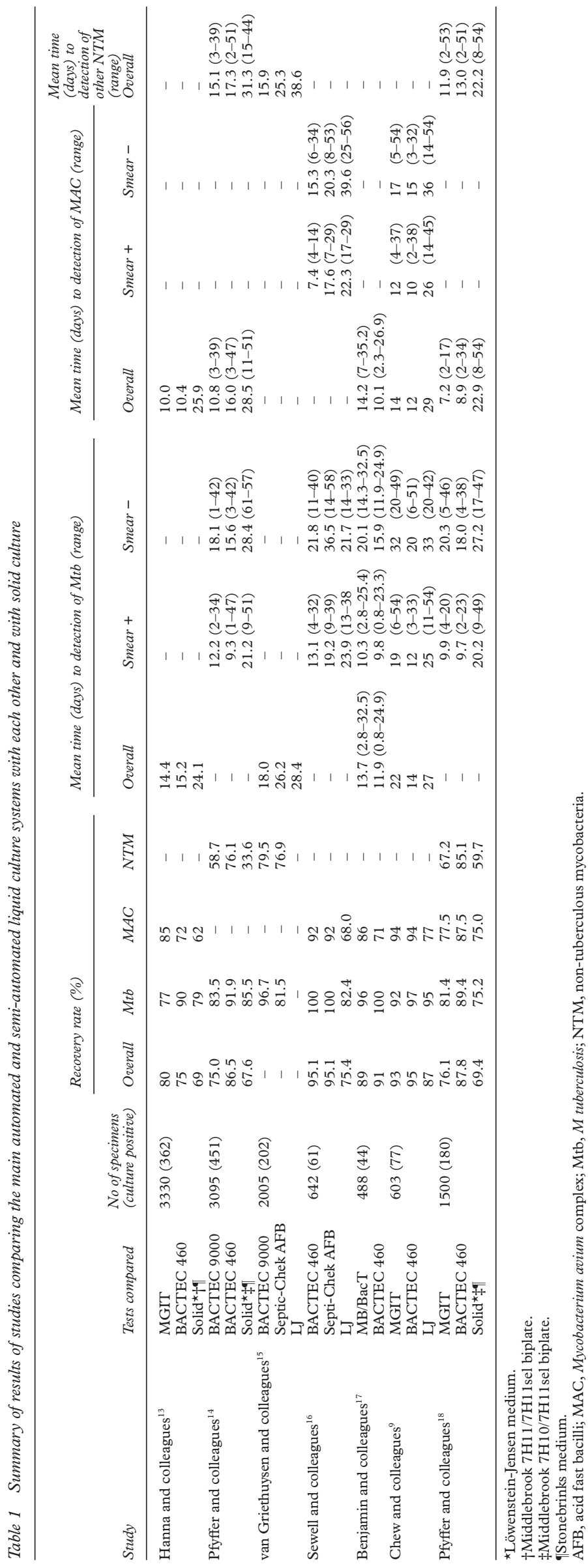

Experience in our laboratory has shown that the algorithm should be standardised in laboratories before use.

We are evaluating a commercially available kit (LiPA MYCOBACTERIA; Innogenetics, Zwijndrecht, Belgium) based on the reverse hybridisation principle. PCR is used to amplify the $16 \mathrm{~S}-23 \mathrm{~S}$ ribosomal spacer region and the product hybridised to probes bound to a nylon strip; binding is identified colorimetrically. Unlike Accuprobe, a single test can identify a range of species with probes specific for the following: Mycobacterium sp., $M$ tuberculosis complex, $M$ kansasii group I, group II, and groups III-V, M xenopi, M gordonae, MAIS group, $M$ avium, $M$ intracellulare, $M$ scrofulaceum, and $M$ chelonae groups I-IV (SA Watterson et al. Utility of the PCR based LiPA MYCOBACTERIA for the identification of mycobacteria isolated in the $\mathrm{MB} / \mathrm{BacT} \mathrm{TB}$ system. Abstracts of the 99th General Meeting of the American Society for Microbiology, abstract no. U-29:639).

An extension of the principle of solid phase detection of nucleic acids has been developed. Using high density DNA probe arrays on a glass "microchip" the system is theoretically capable of simultaneous strain identification, typing, and the detection of drug resistance alleles in cultured isolates. ${ }^{24} 25$ The system has been commercially developed but the cost of specialised analytical equipment is prohibitive.

\section{Rapid methods of detecting mycobacteria} in clinical specimens

As a result of the slow growth of most pathogenic mycobacteria, tests have been developed for the detection of mycobacteria directly in clinical specimens. Most have involved amplification of the small amounts of nucleic acid present, of which the best known is PCR. First described in 1988, it was used to detect $M$ tuberculosis a year later. ${ }^{26}$ Heralded because of a theoretical ability to detect a single copy of a genomic sequence, a decade later a more pragmatic attitude has developed towards its use. Although the specificity of a well developed PCR can be high, the sensitivity is significantly less than that of culture. The sensitivity can be improved by using a nested PCR in which the first round reaction is re-amplified in a second PCR, but the best way to improve sensitivity is to use high quality specimens. Typically, microscopy would be able to detect AFBs in $60-70 \%$ of culture positive respiratory material. The sensitivity of a good quality PCR would be expected to be $90-100 \%$ and $60-70 \%$ on smear positive and negative culture positive respiratory samples, respectively. A positive PCR result using $M$ tuberculosis specific primers on a smear positive sample would indicate that the AFBs present were $M$ tuberculosis, whereas a negative PCR would suggest an NTM, as long as controls were performed to eliminate the possibility of reaction inhibition by sample components. With a smear negative specimen, however, a negative PCR does not exclude the presence of $M$ tuberculosis (see below). 


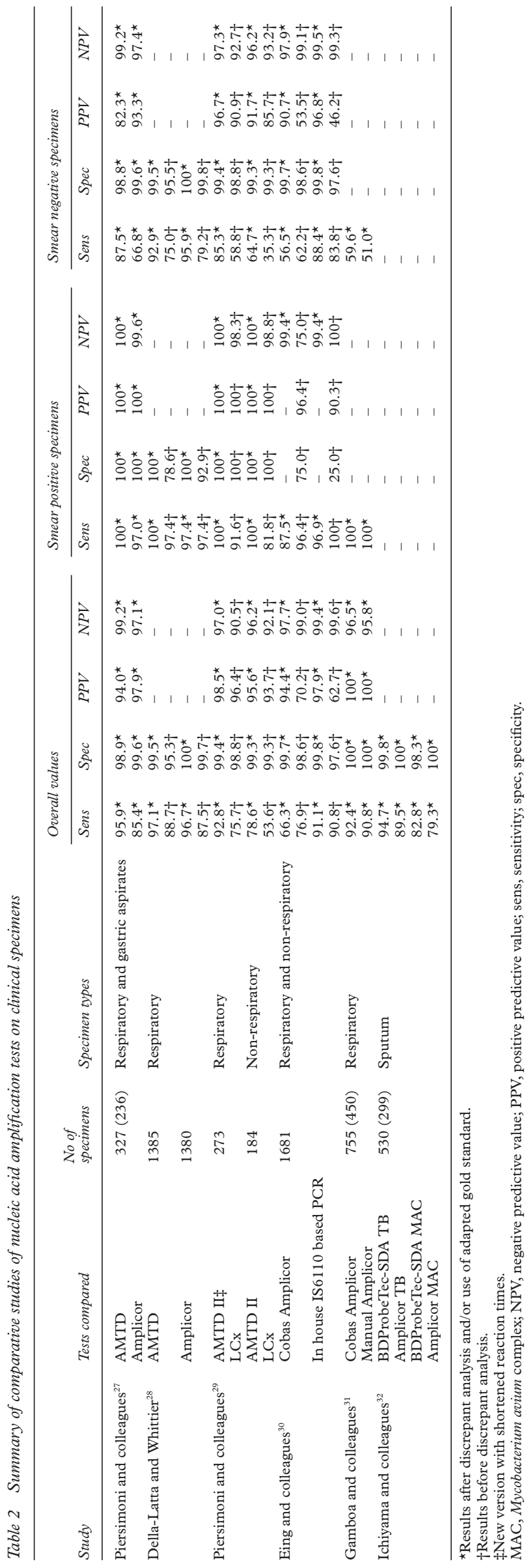

A wide range of alternative nucleic acid amplification tests (NAATs) have been developed, but only a few have been evaluated in the clinical laboratory. Those that have include the PCR based Amplicor (Roche), transcription mediated amplification (AMTD; GenProbe), strand displacement amplification (BDProbeTec-SDA), and the ligase chain reaction (LCx; Abbott Systems). Amplicor amplifies part of the 16S rRNA gene followed by colorimetric detection of the PCR product. The AMTD, rather than using DNA as the target uses rRNA, which is more abundant than the corresponding DNA, making the AMTD theoretically more sensitive than PCR. The rRNA is transcribed by a reverse transcriptase forming a transcription complex from which isothermal amplification of the target sequence is catalysed by RNA polymerase. The BDProbeTec-SDA, like the AMTD, uses isothermal amplification, but uses the Klenow fragment of DNA polymerase I to amplify DNA products from a DNA template. The sensitivity and specificity of such tests have been well established in both single and comparative evaluations. Sensitivity and specificity for most of the above tests fall within the same range of values given (above) for PCR. However, test performances can only be compared if the tests have been performed on the same specimens. Table 2 summarises the main comparative studies. Crossreaction of the GenProbe AMTD test has recently been reported with some isolates of $M$ kansasii and $M$ avium..$^{33}$ It is important to remember that a laboratory report is only as good as the specimen submitted and this is as true for NAATs as it is for any other laboratory test. ${ }^{34} \mathrm{It}$ has been our experience that the key to reliable results from NAAT is the submission of a high quality specimen. In addition, appropriate infrastructure, staff training, and quality control procedures are essential to avoid crosscontamination events leading to false positive results.

\section{Serological tests}

Serological tests for TB are not as well developed as NAATs. Although current tests lack sensitivity, their relatively low cost and simplicity justify further research and development. A comprehensive review of serological tests for TB has been produced by Wilkins. ${ }^{35}$

\section{Rapid methods of detecting drug resistance}

Because the aim of diagnosis is to enable the application of an appropriate treatment regimen, accurate drug susceptibility testing is an intrinsic part of the diagnostic process. Conventional methods can take two weeks to perform when the starting material is a cultured isolate. Rapid methods can be either phenotypic, or genotypic, where screening for drug resistance mutations is performed. Currently, the only drug for which it is practical to screen for resistance mutations clinically is rifampicin because most mutations are confined to a short section of the gene encoding the $\beta$ subunit of the bacterial RNA polymerase. 
Table 3 Decision to treat analysis based on the availability of AFB smear and DAT result

\begin{tabular}{|c|c|c|c|c|c|c|c|c|c|}
\hline \multirow[b]{3}{*}{ Action } & \multirow[b]{3}{*}{$D A T$ result } & \multicolumn{4}{|c|}{ High clinical suspicion of tuberculosis } & \multicolumn{4}{|c|}{ Low clinical suspicion of tuberculosis } \\
\hline & & \multicolumn{2}{|l|}{ Smear positive } & \multicolumn{2}{|l|}{ Smear negative } & \multicolumn{2}{|l|}{ Smear positive } & \multicolumn{2}{|l|}{ Smear negative } \\
\hline & & $\begin{array}{l}\text { Action without } \\
\text { DAT result }\end{array}$ & $\begin{array}{l}\text { Action with } \\
\text { DAT result }\end{array}$ & $\begin{array}{l}\text { Action without } \\
\text { DAT result }\end{array}$ & $\begin{array}{l}\text { Action with } \\
\text { DAT result }\end{array}$ & $\begin{array}{l}\text { Action without } \\
\text { DAT result }\end{array}$ & $\begin{array}{l}\text { Action with } \\
\text { DAT result }\end{array}$ & $\begin{array}{l}\text { Action without } \\
\text { DAT result }\end{array}$ & $\begin{array}{l}\text { Action with } \\
\text { DAT result }\end{array}$ \\
\hline Treat & + & Yes & Yes & Yes & Yes & Yes & Yes & No & ? \\
\hline Treat & - & Yes & $?$ & Yes & $?$ & Yes & No & No & No \\
\hline
\end{tabular}

This table is modified from Catanzaro et al. ${ }^{43}$

Question marks indicate that the clinical, public health, and laboratory groups could not agree on action in these cases, but instead recommend re-assessment of clinical details and repeat testing.

$\mathrm{AFB}$, acid fast bacilli; DAT, direct amplification test.

The InnoLiPA Rif.TB assay ${ }^{36}$ and an RNARNA mismatch assay ${ }^{37}$ have been used successfully in our laboratory for the simultaneous identification of $M$ tuberculosis and screening for rifampicin resistance mutations directly in smear positive respiratory specimens and cultured isolates. In our hands the InnoLiPA Rif.TB and MisMatch assays correlated with standard methods of detection of $M$ tuberculosis and rifampicin susceptibility testing in $94.7 \%$ and $100 \%$ of smear positive respiratory specimens, respectively. ${ }^{38}$

Rapid phenotypic methods include the experimental luciferase phage assay ${ }^{39}$ and the phage amplified biologically (PhaB) assay. ${ }^{38} 40$ These offer attractive advantages over genotypic assays because the mechanism of resistance is irrelevant. Moreover, the technology is substantially cheaper in the case of the PhaB assay, which also requires no expertise beyond that found in any microbiology laboratory. However, to date, although both assays work well on cultured isolates, their application to primary specimens has been more limited. ${ }^{41}$ More detailed descriptions of the $\mathrm{PhaB}$ assay and the genetics of drug resistant tuberculosis can be found in the reviews by Drobniewski and Wilson ${ }^{7}$ and Telenti. ${ }^{42}$

Appropriate use of new technologies in the diagnostic procedure

A 1996 workshop under the auspices of the American Thoracic Society discussed the clinical, laboratory, and public health aspects of direct amplification tests (DATs)amplification tests performed on primary specimens. ${ }^{43}$ The main conclusions of the report were that the tests were a major improvement over standard techniques, although concern was expressed that not enough information existed on their clinical and public health implications. Table 3 summarises the interpretation of DAT results, but where the microscopy and DAT results were discordant the report recommended that further consideration to clinical detail and repeat testing should be performed. The final recommendation was that DATs should always be performed in conjunction with microscopy and culture and that the result should be interpreted alongside the clinical data. Local test performance and underlying TB prevalence are also important variables that will have an impact on the decision analysis of results from DAT assays.

Molecular amplification tests are expensive and no health care system could accommodate their use for every specimen, particularly because novel culture based systems remain more sensitive. Patient management is not always influenced by the test result. Therefore, it makes sense to restrict the use of such tests to situations where clinical management will be assisted by the inclusion of a molecular test. The FDA has approved the AMTD and Amplicor assays for smear positive respiratory specimens from patients not on antibiotic treatment for more than seven days or in the previous 12 months. The tests were not initially approved for smear negative specimens because the number of false positives relative to the extra number of cases detected would have been too high. In most cases the course of disease will be relatively slow and the patient not too unwell. When a patient is critically ill, however, with acute severe disease such as meningitis, it may be desirable to pursue any form of possible rapid diagnosis. Rau and $\operatorname{Libman}^{44}$ performed decision analyses on various strategies for the detection of cases of pulmonary TB. They concluded that PCR on smear positive samples and on media from vials inoculated with smear negative specimens provided shorter average detection times while avoiding the use of DAT as an unrestricted/universal screening tool.

In the UK, mycobacterial isolates are sent to a small number of reference laboratories where identification and drug susceptibility testing is performed. Recent audits have identified several practices that cause delay in diagnosis, namely:

(1) The use of a single solid culture medium for the isolation of mycobacteria from primary specimens instead of a combination of solid and liquid media.

(2) The batching of specimens for processing. All primary specimens should be examined by microscopy and inoculated to culture media within one working day.

(3) Delays in typing of referral forms or the distribution of results to clinicians.

(4) Transcription of reference results to the trust's own IT system. All reference reports should be copied on arrival for later IT entry, with the original reports being immediately transmitted to the clinician. Novel molecular techniques cannot compensate for these delays.

To deal with some of these problems, PHLS laboratories are sending the first smear positive specimen from each patient directly to the Mycobacterium Reference Unit or the 
Regional Centres for Mycobacteriology for rapid culture in an automated system, as already occurs in Scotland. Furthermore, all positive cultures from new patients that resemble $M$ tuberculosis by microscopy and/or macroscopy are identified on the day of receipt. Appropriate susceptibility tests are inoculated immediately. Laboratories are receiving notification of culture receipt and $M$ tuberculosis identification on new patients within 48-72 hours of receipt at no cost to NHS trusts. ${ }^{45}$ Although this is a major improvement, there is a need for all laboratories to identify all possible sources of delay in their $M$ tuberculosis culture and referral systems.

1 Kumar D, Watson JM, Charlett A, et al. Tuberculosis in England and Wales in 1993: results of a national survey. Thorax 1997;52:1060-7.

2 Raviglione MC, Snider DE, Kochi A. Global epidemiology of tuberculosis: morbidity and mortality of a worldwide epidemic. FAMA 1995;273:220-6.

3 Yates MD, Pozniak A, Grange JM. Isolation of mycobacteria from patients seropositive for the human immunofrom patients seropositive for the human immunodeficiency virus (HIV)
Thorax 1993;48:990-5.

4 Ormerod P, Campbell I, Novelli V, et al. Chemotherapy and management of tuberculosis in the United Kingdom: management of tuberculosis in the United King
recommendations 1998. Thorax 1998;53:536-48.

5 American Thoracic Society. Diagnosis and treatment of disease caused by nontuberculous mycobacteria. Am f Respir Crit Care Med 1997;156(suppl):S10-12.

6 Rouillon A, Perdrizet S, Parrot R. Transmission of tubercle bacilli: the effects of chemotherapy. Tubercle 1976;57:27599.

7 Drobniewski FA, Wilson SM. The rapid diagnosis of isoniazid and rifampicin resistance in Mycobacterium
tuberculosis-a molecular story. $\mathcal{F}$ Med Microbiol 1998;47: 189-96.

8 Tenover FC, Crawford JT, Huebner RE et al. The resurgence of tuberculosis: is your laboratory ready? $\mathcal{F}$ Clin Microbiol 1993;31:767-70.

9 Chew WK, Lasaitis RM, Schio FA, et al. Clinical evaluation of the mycobacteria growth indicator tube (MGIT) compared with radiometric (Bactec) and solid media for isolation of mycobacterium species. $\mathcal{F}$ Med Microbiol 1998; 47:821-7.

10 Smithwick RW. 1975. Laboratory manual for acid-fast microscopy, 2nd ed. Atlanta: Center for Disease Control.

11 Damato JJ, Collins MT, Rothlauf MV, et al. Detection of mycobacteria by radiometric and standard plate procedures. F Clin Microbiol 1983;17:1066-73.

12 Stender H, Lund K, Petersen KH, et al. Fluorescence in situ hybridisation assay using peptide nucleic acid probes for differentiation between tuberculous and nontuberculous mycobacterium species in smears of mycobacterium cultures. ₹ Clin Microbiol 1999;37:2760-5.

13 Hanna BA, Ebrahimzadeh A, Elliot LB, et al. Multicenter evaluation of the BACTEC MGIT 960 system for recovery of mycobacteria. F Clin Microbiol 1999;37:748-52.

14 Pfyffer GE, Cieslak C, Welscher H-M, et al. Rapid detection of mycobacteria in clinical specimens by using the
automated BACTEC $9000 \mathrm{MB}$ system and comparison with radiometric and solid-culture systems. F Clin Microbiol with radiometric 4 .

15 van Griethuysen AJ, Jansz AR, Buiting AG. Comparison of fluorescent BACTEC 9000 MB system, Septi-Check AFB system, and Löwenstein-Jensen medium for detection of mycobacteria. F Clin Microbiol 1996;34:2391-4.

16 Sewell DL, Rashad AL, Rourke WJ, et al. Comparison of the Septi-Chek AFB and BACTEC systems and conventional culture for recovery of mycobacteria. F Clin Microbiol 1993; 31:2689-91.

17 Benjamin WH, Waites KB, Beverly A, et al. Comparison of the $\mathrm{MB} / \mathrm{BacT}$ system with a revised antibiotic supplement kit to the BACTEC 460 system for detection of mycobac-
teria in clinical specimens. F Clin Microbiol 1998;36: 3234-8.

18 Pfyffer GE, Welscher H-M, Kissling P, et al. Comparison of the mycobacteria growth indicator tube (MGIT) with radiometric and solid culture for recovery of acid-fast bacilli. F Clin Microbiol 1997;35:364-8.

19 Drobniewski FA, Watt B, Smith EG, et al. A national audit of the laboratory diagnosis of tuberculosis and other myco-
bacterial diseases within the United Kingdom. F Clin Pathol bacterial disease

20 Glickman SE, Kilburn JO, Butler WR, et al. Rapid identification of mycolic acid patterns of mycobacteria by high-performance liquid chromatography using pattern recognition software and a mycobacterium library. $\mathcal{F}$ Clin Microbiol 1994;32:740-5.
21 Kirschner P, Böttger EC. Species identification of mycobacteria using rDNA sequencing. In: Parish T, Stoker NG, eds. Mycobacteria protocols. Totowa, New Jersey: Humana Press, 1998:349-61

22 Telenti A, Marchesi F, Balz M, et al. Rapid identification of mycobacteria to the species level by polymerase chain reaction and restriction enzyme analysis. F Clin Microbiol 1993; 31:175-8.

23 Devallois A, Goh KS, Rastogi N. Rapid identification of mycobacteria to species level by PCR-restriction fragment length polymorphism analysis of the hsp65 gene and proposition of an algorithm to differentiate 34 mycobacterial species. f Clin Microbiol 1997;35:2969-73.

24 Gingeras TR, Ghandour G, Wang E, et al. Simultaneous genotyping and species identification using hybridisation pattern recognition analysis of generic mycobacterium pNA arrays. Genome Res 1998;8:435-48.

25 Troesch A, Nguyen C, Miyada CG, et al. Mycobacterium species identification and rifampin resistance testing with species identification and rifampin resistance testing with
high-density DNA probe arrays. F Clin Microbiol 1999;37: 49-55.

26 Brisson-Noël A, Gicquel B, Lecossier D, et al. Rapid diagnosis of tuberculosis by amplification of mycobacterial DNA in clinical samples. Lancet 1989;4:1069-71.

27 Piersimoni C, Callegaro A, Nista D, et al. Comparative evaluation of two commercial amplification assays for direct detection of Mycobacterium tuberculosis complex in respiratory specimens. F Clin Microbiol 1997;35:193-6.

28 Della-Latta P, Whittier S. Comprehensive evaluation of performance, laboratory application, and clinical usefulness of two direct amplification technologies for the detection of Mycobacterium tuberculosis complex. Am $\mathcal{F}$ Clin Patho 1998;110:301-10.

29 Piersimoni C, Callegaro A, Scarparo C, et al. Comparative evaluation of the new Gen-Probe Mycobacterium tuberculosis amplified direct test and the semiautomated Abbott LCx Mycobacterium tuberculosis assay for direct detection of Mycobacterium tuberculosis complex in respiratory and extrapulmonary specimens. f Clin Microbiol 1998;36: and extrapt $3601-4$.

30 Eing BR, Becker A, Sohns A, et al. Comparison of Roche Cobas Amplicor Mycobacterium tuberculosis assay with in-house PCR and culture for detection of M. tuberculosis. f Clin Microbiol 1996;36:2023-9.

31 Gamboa F, Manterola M, Lonca J, et al. Comparative evaluation of two commercial assays for direct detection of Mycobacterium tuberculosis in respiratory specimens. Eur $\mathcal{7}$ Clin Microbiol Infect Dis 1998;17:151-7.

32 Ichiyama S, Ito Y, Sigiura F, et al. Diagnostic value of the strand displacement amplification method compared to those of Roche Amplicor PCR and culture for detecting mycobacteria in sputum samples. $\mathcal{F}$ Clin Microbiol 1997;35: 3082-5.

33 Jorgensen JH, Salinas JR, Paxson R, et al. False-positive Gen-Probe direct Mycobacterium tuberculosis amplification test results for patients with pulmonary M. kansasii and M. avium infections. F Clin Microbiol 1999;37:175-8.

34 Heifets LB, Good RB. Current laboratory methods for the diagnosis of tuberculosis. In: Bloom BR, ed. Tuberculosis, diagnosis of tuberculosis. In: Bloom BR, ed. Tuberculosis,
pathogenesis, protection and control. Washington, DC: Ameripathogenesis, protection and control. Washington,

35 Wilkins EGL. Antibody detection in tuberculosis. In: Davies PDO, ed. Clinical tuberculosis, 2nd ed. London: Chapman and Hall, 1998:81-95.

36 Rossau R, Traore H, De Beenhouwer $\mathrm{H}$, et al. Evaluation of the INNO-LiPA Rif.TB assay, a reverse hybridisation assay for the simultaneous detection of Mycobacterium tuberculosis complex and its resistance to rifampin. Antimicrob Agents Chemother 1997;41:2093-8.

37 Nash KA, Gaytan A, Inderlied CB. Detection of rifampin resistance in Mycobacterium tuberculosis by means of a rapid, simple, and specific RNA/RNA mismatch assay. $\mathcal{f}$ Infect Dis 1997;176:533-6.

38 Watterson SA, Wilson SM, Yates MD, et al. Comparison of three molecular assays for the rapid detection of rifampin three molecular assays for the rapid detection of rifampin 1998;36:1969-73.

39 Riska PF, Su Y, Bardarov S, et al. Rapid film-based determination of antibiotic susceptibilities of Mycobacterium tuberculosis strains by using a luciferase reporter phage and the Bronx Box. F Clin Microbiol 1999;37:1144-9.

40 Wilson SM, Al-Suwaidi Z, McNerney R, et al. Evaluation of a new rapid bacteriophage-based method for the drug susceptibility testing of Mycobacterium tuberculosis. Nat Med 1997;3:465-8

41 Drobniewski FA, Wilson SM. New biomolecular assays must be tested by direct study in the developing world. $B M \mathcal{F}$ 1998;316:940

42 Telenti A. Genetics of drug resistant tuberculosis. Thorax 1998;53:793-7.

43 Catanzaro A, Davidson BL, Fujiwara PI, et al. Rapid diagnostic tests for tuberculosis. What is the appropriate diagnostic tests for tuberculosis. What is the approp
use? Am $\mathcal{7}$ Respir Crit Care Med 1997;155:1804-14.

44 Rau NV, Libman MD. Laboratory implementation of the polymerase chain reaction for confirmation of pulmonary tuberculosis. Eur f Clin Microbiol Infect Dis 1999;18:35-41.
Drobniewski FA. Diagnosing multidrug resistant tuberculosis in Britian. Clinical suspicion should drive rapid diagnosis. $B M F 1998 ; 317: 1263-4$ 